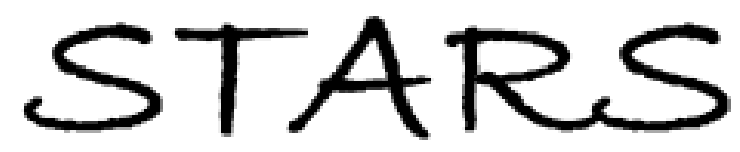

University of Central Florida

STARS

$1-1-1997$

\title{
Ab initio calculation for low-energy elastic scattering of electrons from sulfur atoms
}

H. P. Saha

University of Central Florida

Dong Lin

University of Central Florida

Find similar works at: https://stars.library.ucf.edu/facultybib1990

University of Central Florida Libraries http://library.ucf.edu

This Article is brought to you for free and open access by the Faculty Bibliography at STARS. It has been accepted for inclusion in Faculty Bibliography 1990s by an authorized administrator of STARS. For more information, please contact STARS@ucf.edu.

\section{Recommended Citation}

Saha, H. P. and Lin, Dong, "Ab initio calculation for low-energy elastic scattering of electrons from sulfur atoms" (1997). Faculty Bibliography 1990s. 2084.

https://stars.library.ucf.edu/facultybib1990/2084

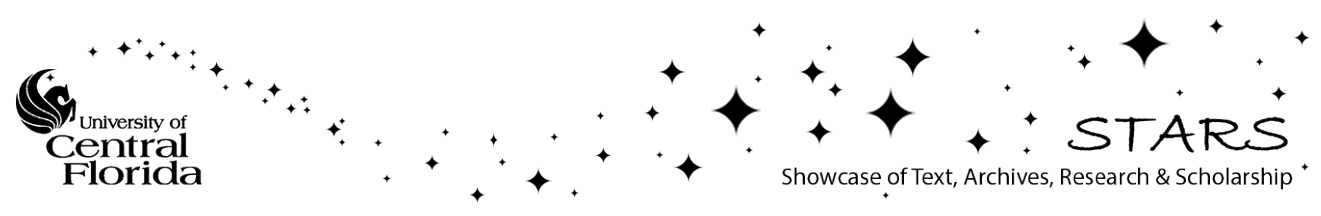




\title{
$A b$ initio calculation for low-energy elastic scattering of electrons from sulfur atoms
}

\author{
H. P. Saha and Dong Lin \\ Department of Physics, University of Central Florida, Orlando, Florida 32816
}

(Received 25 November 1996; revised manuscript received 12 March 1997)

\begin{abstract}
The multiconfiguration Hartree-Fock method for continuum wave functions has been used to perform calculations of elastic scattering of electrons from the open-shell sulfur atom at low energies. The electron correlation and polarization of the sulfur atom by the scattered electron, which are very important in this calculation, have been taken into account very accurately $a b$ initio through the configuration-interaction procedure by optimizing both bound and continuum orbitals simultaneously at each kinetic energy of the scattered electron. The scattering lengths, phase shifts, and differential, total and momentum-transfer cross sections for electrons elastically scattered from sulfur atoms are calculated for the impact-energy range from 0 to $27.2 \mathrm{eV}$. The wave functions computed exactly at zero energy are used to compute the scattering length. The present results are compared with other available theoretical results. [S1050-2947(97)08208-5]
\end{abstract}

PACS number(s): 34.80.-i, 32.80.Bx

\section{INTRODUCTION}

For a long time the study of scattering of electrons by closed-shell atoms has been the subject of extensive investigation both theoretically and experimentally. Conversely, scattering of electrons from open-shell atoms is much more difficult to calculate because of strong correlation within the target states of the atom. In particular, elastic scattering of electrons from open-shell atoms presents a challenge to the theorist because of the large polarization and electron correlation effects on the target atom by the scattered electrons, especially at very low collision energy. Recently, considerable interest has developed in understanding the details of electron scattering from open-shell atoms because of its application to low-temperature astrophysical plasmas and also to plasma processing of semiconductors.

Here we present the results of calculations on elastic scattering of electrons from the open-shell sulfur atom at low collision energies. As far as we are aware, there is no experimental data available on this process. In the literature there is only one theoretical calculation reported for electron energies below $1.0 \mathrm{eV}$. This calculation was performed by Fabricant [1] using the method of extrapolation of potential parameters along isoelectronic series and among corresponding neutral atoms. The presence of a Ramsauer-Townsend minimum in the total and momentum-transfer cross sections makes this $e^{-}+\mathrm{S}$ calculation more interesting from the theoretical point of view. In the case of closed-shell atoms, e.g., the inert gases, Ramsauer-Townsend minima are observed [2-7] in the total and momentum-transfer cross sections of elastic scattering of electrons from argon, krypton, and xenon atoms. In the case of elastic scattering of electrons from chlorine atoms investigated recently [8], we also found a Ramsauer-Townsend minimum at very low energies. It is important as well as interesting to investigate what other elements below argon in the periodic table show this important feature.

We have seen that the multiconfiguration Hartree-Fock (MCHF) method for continuum-wave functions is capable of producing accurate data for scattering lengths, phase shifts, and total, momentum-transfer, and differential cross sections for elastic scattering of electrons from noble-gas atoms at very low energies. In these calculations, the important electron correlation and polarization effects are taken into account by optimizing both excited bound orbitals and continuum orbitals at each kinetic energy of the scattered electron through the configuration-interaction procedure. It is found that the MCHF procedure calculates these effects more accurately than any other method at low energies. The results of these calculations were found to be in excellent agreement with accurate experimental and other theoretical results $[4,5]$.

In this paper we present results of our calculation of elastic scattering of electrons from the ground $3 s^{2} 3 p^{4}{ }^{3} P$ state of sulfur atoms using the multiconfiguration Hartree-Fock (MCHF) method for continuum-wave functions [9]. We hope this calculation will encourage experimentalists to measure cross sections for this important process.

The elastic scattering of electrons from sulfur atoms is not only different from the closed-shell noble-gas atoms, but it is also different from the open-shell chlorine atom because its ground-state configuration produces three target states whose coupling with the incoming electron is very strong. The main purpose of this investigation is not only to see how accurately the scattering lengths and cross sections in the case of electron-sulfur scattering can be obtained using the MCHF method, but also to determine the differences between the closed-shell and the open-shell atoms with regard to scattering length and the Ramsauer-Townsend minimum. We have calculated completely ab initio, the scattering lengths, and differential, integral, and momentum-transfer cross sections through an energy range from 0 to $27.2 \mathrm{eV}$, an extremely difficult region for an $a b$ initio calculation. As the polarization of the target by the projectile depends on the kinetic energy of the projectile, and is different for different kinetic energies, in the MCHF approach the polarization effects are considered through the bound configurations, which represent the multipole polarization, by varying the excited bound orbitals and the continuum orbitals simultaneously at each kinetic energy of the projectile. The differential, total elastic, and momentum-transfer cross sections are calculated with the computed scattering length and the phase shifts obtained by the MCHF method. We shall assume that the spin-orbit 
interaction and other relativistic effects are not significant and that the $L S$ coupling calculation is adequate in this elastic scattering of electrons from neutral sulfur. The only theoretical results with which we compare our results are also nonrelativistic.

\section{THEORY}

\section{A. MCHF wave function for a scattering state}

The wave function for a scattering state with label $\gamma$, energy $E$, and term $L S$, in the multiconfiguration Hartree-Fock approximation can be expressed as

$$
\begin{aligned}
\Psi(\gamma L S ; N+1)= & \sum_{j}^{m_{t}} a_{j} \Phi\left(\gamma_{j} L_{t} S_{t} ; N\right) \phi_{k l} \\
& +\sum_{i}^{m} c_{i} \Phi\left(\gamma_{i} L S ; N+1\right),
\end{aligned}
$$

where the first term represents a correlated target wave function describing an $N$-electron target that is an eigenstate of $L_{t}$ and $S_{t}$ in terms of $N$-electron bound configuration states $\Phi\left(\gamma_{j} L_{t} S_{t} ; N\right)$ with configuration $\gamma_{j}$ and term $L_{t} S_{t}$, mixing coefficients $a_{j}$, and total energy $E_{t}$ coupled to a scattering wave function $\phi_{k l}$ with orbital angular momentum $l$, to yield an antisymmetric configuration state for the $(N+1)$-electron system, with final term value $L S$ and configuration $\gamma_{j} k l$. In the second term $\Phi\left(\gamma_{i} L S ; N+1\right)$ are $(N$ $+1)$-electron bound configurations which are eigenstates with the same $L$ and $S$ and which are included to allow for polarization and electron-correlation effects.

The above wave function is defined in terms of a set of radial functions $P_{i}(r), i=1,2, \ldots, m$. Briefly, the MCHF method optimizes both bound and continuum-wave functions simultaneously at each incident energy. The optimized bound orbitals take into account the polarization and correlation effects very accurately, resulting in an accurate phase shift. The MCHF method for scattering wave functions is used to calculate the scattering length for zero energy and phase shifts $\delta_{l}$ for various partial waves for low energies. The method of constructing the scattering length and the phase shifts for various partial waves involves the solution of coupled integro-differential equations for the radial functions of the form

$$
\begin{aligned}
{\left[\frac{d^{2}}{d r^{2}}+\frac{2 Z}{r}-\frac{l_{i}\left(l_{i}+1\right)}{r^{2}}\right] P_{i}(r)=} & \frac{2}{r}\left[Y_{i}(r) P_{i}(r)+X_{i}(r)\right. \\
& \left.+I_{i}(r)\right]+\sum_{i^{\prime}} \varepsilon_{i i^{\prime}} P_{i^{\prime}}(r)
\end{aligned}
$$

where $(2 / r) Y_{i}(r)$ is the direct potential, $(2 / r) X_{i}(r)$ is the exchange function, and $(2 / r) I_{i}(r)$ represents terms arising from interactions between configuration states. The offdiagonal energy parameters $\varepsilon_{i i}$, are related to Lagrange multipliers that ensure orthogonality of the wave functions. These equations are solved by an iterative method with the boundary conditions for the bound radial functions

$$
P_{i}(r) \underset{r \rightarrow 0}{\sim} r^{l+1}, \quad P_{i}(r) \underset{r \rightarrow \infty}{\sim} 0
$$

and with those for scattering wave functions

$$
P_{i}(r) \underset{r \rightarrow 0}{\sim} r^{l+1}, \quad P_{i}(r) \underset{r \rightarrow \infty}{\sim} C \sin \left(k r-\frac{l \pi}{2}+\delta_{l}\right),
$$

where $\delta_{l}$ is the phase shift and $\varepsilon_{i i^{\prime}}=-k^{2}, k^{2}$ being the kinetic energy of the scattered electron in rydbergs. For $k=0$ and at large $r$, the scattering wave function for the $s$-partial wave satisfies the condition

$$
P_{i}(r)=A(r-a)
$$

where $a$ is the scattering length and $A$ is the amplitude. The value of $a$ can be calculated directly using the explicit zeroenergy wave function.

To determine the bound and the scattering radial functions, the above set of coupled second-order integrodifferential equations are solved under the proper boundary conditions. The normalization of the scattering radial function is calculated by fitting the computed values at two adjacent points to the regular and irregular Bessel functions as soon as the region where the direct and exchange potentials vanish is reached, which may be at considerably smaller values of $r$ than the asymptotic form given by the boundary condition of Eq. (4).

\section{B. Elastic scattering for open-shell atoms}

The differential cross section $\sigma(\theta)$ for the process $\gamma \rightarrow \gamma^{\prime}$ in atomic units $\left(a_{0}^{2} / \mathrm{sr}\right)$ is given by $[10,11]$

$$
\frac{d \sigma}{d \Omega}=\frac{1}{2\left(2 L_{\gamma}+1\right)\left(2 S_{\gamma}+1\right)} \sum_{\mu^{\prime}} \sum_{\mu}\left|\left(\gamma^{\prime} \mu^{\prime}|f| \gamma \mu\right)\right|^{2},
$$

where

$$
\begin{aligned}
\left(\gamma^{\prime} \mu^{\prime}|f| \gamma \mu\right)= & \sum_{L S \pi} \sum_{l l^{\prime}}\left[\left(2 l^{\prime}+1\right)(2 l+1)\right]^{1 / 2} \\
& \times l^{l-l^{\prime}} d_{m^{\prime} 0}^{l^{\prime}}(\theta) e^{i m^{\prime} \phi}\left(T_{\gamma l^{\prime}, \gamma l^{\prime}}^{L S} / k_{\gamma}\right) \\
& \times\left(L_{\gamma^{\prime}} M_{L \gamma^{\prime}} l^{\prime} m^{\prime} \mid L M_{L}\right)\left(L_{\gamma} M_{L \gamma} l 0 \mid L M_{L}\right) \\
& \times\left(S_{\gamma^{\prime}} M_{S \gamma^{\prime}} \frac{1}{2} m_{S}^{\prime} \mid S M_{S}\right)\left(S_{\gamma} M_{S \gamma^{\frac{1}{2}}} m_{S} \mid S M_{S}\right)
\end{aligned}
$$

and

$$
d_{m^{\prime} 0}^{l^{\prime}}(\theta) e^{i m^{\prime} \phi}=\left(4 \pi / 2 l^{\prime}+1\right)^{1 / 2} Y_{l^{\prime} m^{\prime}}(\theta \phi) .
$$

Explicit formulas for the sums appearing in Eq. (7), expressed in compact form in terms of angular-momentum recoupling coefficients, are given by Blatt and Biedenharn [12]. The final result can be written most simply in terms of the quantities $Z\left(l_{1} L_{1} l_{2} L_{2}, L_{\gamma} J\right)$ [13] defined as follows: 


$$
\begin{aligned}
Z\left(l_{1} L_{1} l_{2} L_{2}, L_{\gamma} J\right)= & \left(2 l_{1}+1\right)^{1 / 2}\left(2 l_{2}+1\right)^{1 / 2}\left(2 L_{1}+1\right)^{1 / 2} \\
& \times\left(2 L_{2}+1\right)^{1 / 2} i^{J-l_{1}+l_{2}} W\left(l_{1} L_{1} l_{2} L_{2}, L_{\gamma} J\right) \\
& \times\left(l_{1} l_{2} 00 \mid J 0\right),
\end{aligned}
$$

where $W$ is the Racah coefficient defined in reference [13]. The properties of $W$ are discussed there. The factor $i^{J-l_{1}+l_{2}}$ is either +1 or -1 , and is never imaginary.

The differential cross section is then given by

$$
\begin{aligned}
\frac{d \sigma}{d \Omega}= & \frac{1}{2\left(2 L_{\gamma}+1\right)\left(2 S_{\gamma}+1\right) k_{\gamma}^{2}} \sum_{S}(2 S+1) \\
& \times \sum_{J=0}^{\infty} B_{J}\left(\alpha^{\prime} \gamma^{\prime}, \alpha \gamma\right) P_{J}(\cos \theta),
\end{aligned}
$$

where

$$
\begin{aligned}
& B_{J}\left(\alpha^{\prime} \gamma^{\prime}, \alpha \gamma\right) \\
& =\frac{(-1)^{L_{\gamma}^{\prime}-L_{\gamma}}}{4} \sum_{L_{1}} \sum_{L_{2}} \sum_{l_{1}} \sum_{l_{2}} \sum_{l_{1}^{\prime}} \sum_{l_{2}^{\prime}} Z\left(l_{1} L_{1} l_{2} L_{2}, L_{\gamma} J\right) \\
& \times Z\left(l_{1}^{\prime} L_{1} l_{2}^{\prime} L_{2}, L_{\gamma}^{\prime} J\right) \\
& \times \operatorname{Re}\left[\left(\delta_{\alpha^{\prime} \alpha} \delta_{L_{\gamma}^{\prime} L_{\gamma}} \delta_{l_{1}^{\prime} l_{1}}-S_{\alpha^{\prime} L_{\gamma}^{\prime} l_{1}^{\prime} ; \alpha L_{\gamma} l_{1}}^{L_{1}}\right)^{*}\right. \\
& \left.\times\left(\delta_{\alpha^{\prime} \alpha} \delta_{L_{\gamma}^{\prime} L_{\gamma}} \delta_{l_{2}^{\prime} l_{2}}-S_{\alpha^{\prime} L_{\gamma}^{\prime} l_{2}^{\prime} ; \alpha L_{\gamma_{2}} l_{2}}^{L_{2}}\right)\right] .
\end{aligned}
$$

For pure elastic scattering, $\alpha^{\prime}=\alpha, L_{\gamma}=L_{\gamma}^{\prime}, l_{1}=l_{1}^{\prime}, l_{2}$ $=l_{2}^{\prime}$ and using $S_{\alpha L_{\gamma} l ; \alpha L_{\gamma} l}^{L}=\exp \left(2 i \delta_{l L S}\right)$, Eq. (11) simplifies to

$$
\begin{aligned}
B_{J}(\alpha \gamma)= & \frac{1}{4} \sum_{L_{1}} \sum_{L_{2}} \sum_{l_{1}} \sum_{l_{2}} Z\left(l_{1} L_{1} l_{2} L_{2}, L_{\gamma} J\right)^{2} \\
& \times \operatorname{Re}\left[\left(1-\exp \left(2 i \delta_{l_{1} L_{1} S}\right)^{*}\left(1-\exp \left(2 i \delta_{l_{2} L_{2} S}\right)\right] .\right.\right.
\end{aligned}
$$

Then the total cross section in units of $a_{0}^{2}$ is

$$
\sigma_{T}=\frac{2 \pi}{\left(2 L_{\gamma}+1\right)\left(2 S_{\gamma}+1\right) k_{\gamma}^{2}} \sum_{S}(2 S+1) B_{0}(\alpha \gamma),
$$

and the momentum-transfer cross section in units of $a_{0}^{2}$ is given by

$$
\begin{aligned}
\sigma_{M}= & \frac{2 \pi}{\left(2 L_{\gamma}+1\right)\left(2 S_{\gamma}+1\right) k_{\gamma}^{2}} \sum_{S}(2 S+1) \\
& \times\left(B_{0}(\alpha \gamma)-\frac{1}{3} B_{1}(\alpha \gamma)\right) .
\end{aligned}
$$

\section{COMPUTATIONAL PROCEDURE}

First of all, the ground-state wave function of the sulfur atom $3 s^{2} 3 p^{4}{ }^{3} P$ is calculated in the Hartree-Fock (HF) approximation [14]. The correlated wave function of the target is then calculated by the multiconfiguration Hartree-Fock wave-function expansion over the configuration states coupled to form a ${ }^{3} P$ term. These configurations are gener- ated by the single and double replacements of the two outermost orbitals $3 s$ and $3 p$ of the sulfur atom by the excited orbitals $3 d, 4 s, 4 p, 4 d, 4 f, 5 s, 5 p, 5 g, 6 h, 7 i$, and $8 k$. The eigenenergy of the ground state of the sulfur atom was found to be -397.6567 a.u. These wave functions are used as an input in the calculation of the scattering wave functions and the phase shifts for various partial waves and various final $L S$ states.

As both polarization and electron-correlation of the openshell $3 s^{2} 3 p^{4}$ target atom by the scattering electron are very important at zero and at very low-energy $e^{-}+\mathrm{S}$ scattering, these have been taken into account very accurately through the configuration interaction procedure. Polarization is nothing more than the distortion of the $3 s$ and $3 p$ orbitals of the sulfur atom due to the presence of the electric field of the scattering electron. It is found that dipole polarization alone of the target is sufficient for this case of electron-sulfur scattering. The bound configurations which will account for the dipole polarization of the $3 s$ and $3 p$ target orbitals are generated by the replacements $3 s \rightarrow n p, n \leqslant 5 ; 3 p \rightarrow n d, n \leqslant 4$; and $3 p \rightarrow n s, n \leqslant 5$. All the configurations generated in this way are considered in the expansion of the scattering wave functions. These excited bound orbitals $n s, n p$, and $n d$ are determined by optimizing both the excited bound orbitals and the scattering electron orbitals $k l$ simultaneously at each kinetic energy of the incident electron. This procedure includes the dynamical polarization of the target more accurately in the ab initio way. The same set of configurations, representing the polarization and electron correlation effects, are used for each partial wave and each final state to calculate the scattering wave functions over the range of energies 0-27.2 eV.

Partial waves $l=0-6$ are calculated directly by the MCHF method. The effective range formula [15]

$$
\tan \delta_{l}=\frac{\pi \alpha k^{2}}{(2 l+3)(2 l+1)(2 l-1)},
$$

is used to calculate partial-wave contributions for $l>6$. In this expression $\alpha$ is the static dipole polarizability of the target atom. The dipole polarizability $[16,17]$ obtained in this calculation with the multiconfiguration wave functions is $19.44 a_{0}^{3}$, which agrees very well with the experimental value of $19.6 a_{0}^{3}$ [18]. Since the contributions to the cross section from the higher partial waves in the energy range considered are, in general, quite small, the effective range formula (14) provides reasonably accurate higher partial-wave phase shifts.

\section{RESULTS}

In the present paper, we performed detailed calculations to determine the effects of polarization and electron correlation on the low-energy elastic scattering of electrons from sulfur atoms. The scattering lengths, phase shifts, differential, integral elastic, and momentum-transfer cross sections are calculated for impact energies from 0 to $27.2 \mathrm{eV}$.

\section{A. Phase shifts}

We calculated phase shifts for each $l$ and $L S$ term of the resultant configuration in the MCHF approximation. In the present calculation there are $38 L S$ terms for $l=0-6$. In Table I, we present phase shifts for selected energies for $l$ 
TABLE I. Phase shifts $\delta_{l}$ for sulfur.

\begin{tabular}{|c|c|c|c|c|c|c|c|}
\hline $\begin{array}{l}\text { Energy } \\
k^{2} \text { (Ry) }\end{array}$ & $\delta_{0}\left({ }^{2} P\right)$ & $\delta_{1}\left({ }^{2} D\right)$ & $\delta_{2}\left({ }^{2} F\right)$ & $\delta_{3}\left({ }^{2} G\right)$ & $\delta_{4}\left({ }^{2} H\right)$ & $\delta_{5}\left({ }^{2} I\right)$ & $\delta_{6}\left({ }^{2} K\right)$ \\
\hline 0.005 & 0.019013 & 0.010137 & -0.000855 & -0.000695 & -0.000712 & -0.000502 & -0.000192 \\
\hline 0.01 & -0.012989 & 0.019542 & 0.000283 & -0.000370 & -0.000747 & -0.000933 & -0.000099 \\
\hline 0.05 & -0.232506 & 0.047097 & 0.015544 & 0.005114 & 0.001393 & -0.000171 & 0.001533 \\
\hline 0.10 & -0.430664 & 0.021434 & 0.043561 & 0.015479 & 0.004388 & 0.002561 & 0.002985 \\
\hline 0.15 & -0.583260 & -0.036497 & 0.083031 & 0.023256 & 0.010047 & 0.005286 & 0.004458 \\
\hline 0.20 & -0.711341 & -0.103807 & 0.128507 & 0.034445 & 0.014551 & 0.007352 & 0.005860 \\
\hline 0.25 & -0.822906 & -0.176508 & 0.189191 & 0.044135 & 0.019083 & 0.010760 & 0.007276 \\
\hline 0.30 & -0.925050 & -0.245662 & 0.259842 & 0.053149 & 0.023375 & 0.013676 & 0.008716 \\
\hline 0.35 & -1.007405 & -0.305615 & 0.332274 & 0.063193 & 0.026945 & 0.015466 & 0.011046 \\
\hline 0.40 & -1.095456 & -0.375858 & 0.412762 & 0.076141 & 0.030805 & 0.017777 & 0.012527 \\
\hline 0.45 & -1.156402 & -0.434218 & 0.499956 & 0.086876 & 0.034619 & 0.020171 & 0.013011 \\
\hline 0.50 & -1.238302 & -0.493644 & 0.589314 & 0.096514 & 0.039048 & 0.022846 & 0.014456 \\
\hline 0.60 & -1.356881 & -0.598166 & 0.765994 & 0.113953 & 0.048529 & 0.028047 & 0.017427 \\
\hline 0.80 & -1.565916 & -0.773972 & 1.076953 & 0.153935 & 0.063355 & 0.037659 & 0.023069 \\
\hline 1.00 & -1.728533 & -0.920450 & 1.308655 & 0.207692 & 0.079484 & 0.047488 & 0.028918 \\
\hline 1.20 & -1.873219 & -1.044714 & 1.508783 & 0.263900 & 0.091036 & 0.055864 & 0.034644 \\
\hline 1.40 & -1.991672 & -1.134653 & 1.657023 & 0.323612 & 0.102902 & 0.064575 & 0.040385 \\
\hline 1.60 & -2.087618 & -1.209929 & 1.809660 & 0.413790 & 0.112365 & 0.073804 & 0.046124 \\
\hline 1.80 & -2.152969 & -1.247031 & 1.983956 & 0.522413 & 0.122596 & 0.084685 & 0.051865 \\
\hline 2.00 & -2.216402 & -1.285501 & 2.167814 & 0.627330 & 0.131256 & 0.093767 & 0.057473 \\
\hline
\end{tabular}

$=0-6$ and for a few doublet $L S$ terms. The results for other $L S$ terms are available on request.

\section{B. Scattering length}

As already mentioned, Fabricant [1] carried out calculations on elastic scattering of electrons from sulfur by the method of extrapolation of potential parameters along the isoelectronic series of positive ions and the corresponding neutral atoms. He calculated the scattering length from the initial $\mathrm{S}\left({ }^{3} P_{2}\right)$ target state and obtained for the final ${ }^{4} P_{3 / 2}$ state a value of -2.60 and for the final ${ }^{2} P_{3 / 2}$ state a value of -1.17 . We performed calculations in $L S$ coupling for both quartet and doublet scattering lengths in both the HartreeFock and MCHF approximations. The results obtained in the HF approximation are $1.6043 a_{0}$ and $2.3209 a_{0}$ for the quartet and doublet terms, respectively, whereas in the MCHF approximation they are $-4.2558 a_{0}$ and $-1.6273 a_{0}$, respec- tively. It will be noticed that the sign of the scattering lengths in the HF approximation is completely reversed in the MCHF approximation. This is due to the effects of polarization and electron correlation. At zero energy, in the HF approximation, the potential is repulsive and as a result the scattering length is positive, but as soon as the polarization and electron correlation effects are taken into account, the potential becomes attractive, which makes the scattering length negative. It should be mentioned that the scattering lengths for scattering of electrons from inert-gas atoms, e.g., helium [19] and neon [20], are positive, indicating that the potentials are repulsive, whereas for argon [2] the scattering length is negative due to an attractive potential similar to that in the present case. In Table II, the rate of convergence for both the quartet and doublet scattering lengths with respect to the number of configurations generated by the orbitals representing multipole polarization is shown. It is found that the scattering length converges very well.

TABLE II. Convergence rate for the scattering length (in units of $a_{0}$ ) with respect to the number of configurations generated by the orbitals representing multipole polarization.

\begin{tabular}{lccc}
\hline \hline$L S$ & Polarized orbitals & Number of configurations & Scattering length \\
\hline Quartet & $3 d, 4 s, 4 p$ & 19 & -2.8973 \\
& $+4 d, 5 s, 5 p$ & 71 & -3.7290 \\
& $+4 f$ & 87 & -4.2248 \\
& $+5 d$ & 101 & -4.2557 \\
& $+6 s$ & 109 & -4.2558 \\
Doublet & $3 d, 4 s, 4 p$ & 26 & -0.9104 \\
& $+4 d, 5 s, 5 p$ & 98 & -1.3709 \\
& $+4 f$ & 124 & -1.6069 \\
& $+5 d$ & 156 & -1.6273 \\
& $+6 s$ & 166 & -1.6273 \\
\hline \hline
\end{tabular}




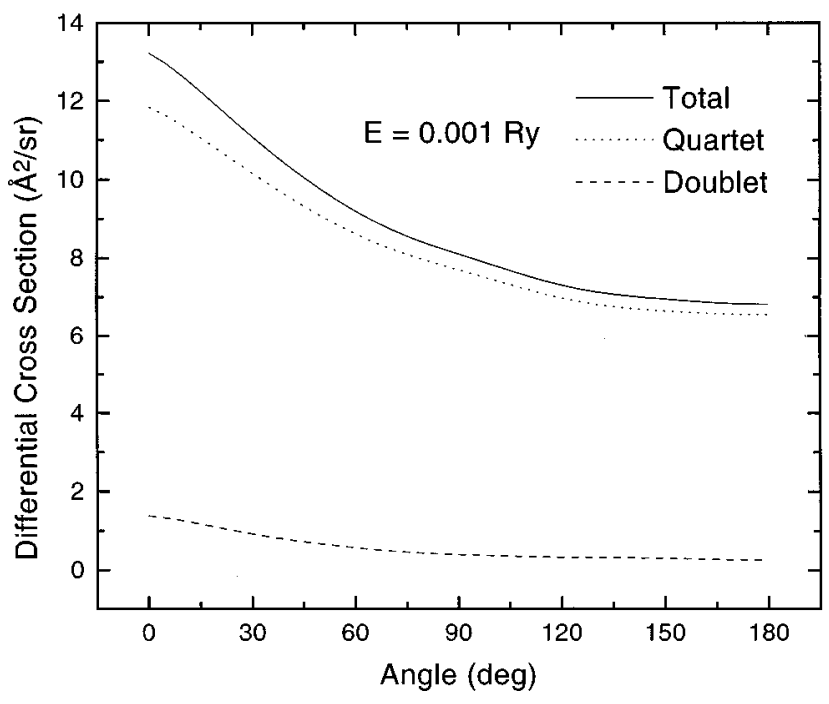

FIG. 1. Differential cross section for elastic scattering of electrons from sulfur at energy $E=0.001 \mathrm{Ry}$ as a function of scattering angle.

\section{Ramsauer minimum}

The most interesting feature, the Ramsauer minimum, is found in the cross section of this $e^{-}-\mathrm{S}$ elastic scattering. The position and magnitude of the Ramsauer-Townsend minimum obtained by the MCHF method are, respectively, $0.476 \mathrm{eV}$ and $5.4439 \AA^{2}$, whereas those obtained by Fabricant [1] are $0.175 \mathrm{eV}$ and about $5.6 \AA^{2}$, respectively. The present minimum occurs at higher energy than that obtained by Fabricant; nevertheless, the magnitude of the minimum we have obtained compares very well with that obtained by Fabricant. This minimum occurs because the attractive potential near this energy is sufficiently strong to insure that the $s$ wave is pulled in by almost half a cycle, and the phase shift $\delta_{0}$ at $l=0$ is almost $\pi$ radians. In this situation the energy of the electron is very small and the attractive potential is very strong so that all other phase shifts are small when $\delta_{0}=\pi$.

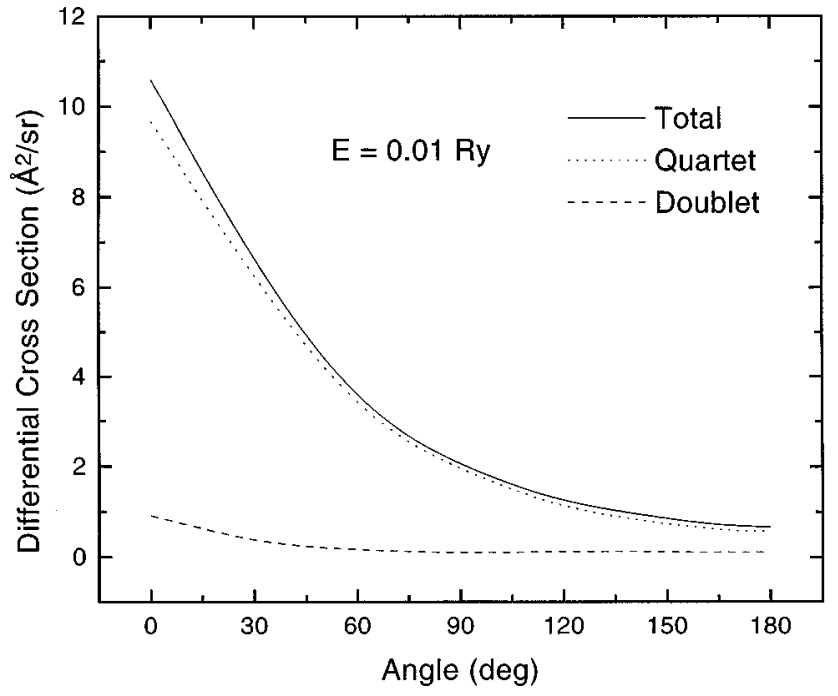

FIG. 2. Differential cross section for elastic scattering of electrons from sulfur at energy $E=0.01 \mathrm{Ry}$ as a function of scattering angle.

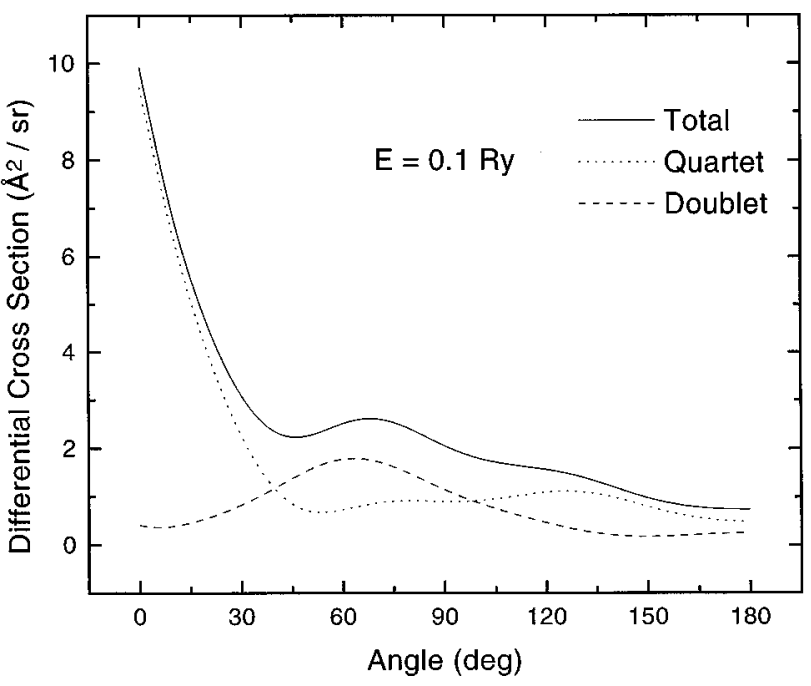

FIG. 3. Differential cross section for elastic scattering of electrons from sulfur at energy $E=0.1 \mathrm{Ry}$ as a function of scattering angle.

As a result, the scattering amplitude $f(\theta)$ becomes very small for all $\theta$, and there is a minimum in the cross section.

\section{Differential cross section}

The differential cross sections computed from the present calculated phase shifts obtained in the MCHF approximation for $k^{2}=0.001,0.01,0.1,1.0 \mathrm{Ry}$ are presented in Figs. 1-4. In each figure both doublet and quartet contributions, together with the total, are plotted as a function of the scattering angle in degrees. As mentioned earlier, the phase shifts for $l=0-6$ have been calculated directly by the MCHF method. The contributions of phase shifts for higher partial waves have been calculated using the effective range formula (14).

For energies 0.001 and $0.01 \mathrm{Ry}$, as shown in Figs. 1 and 2 , respectively, all three cross sections, quartet, doublet and total, decrease with increasing scattering angles. At these en-

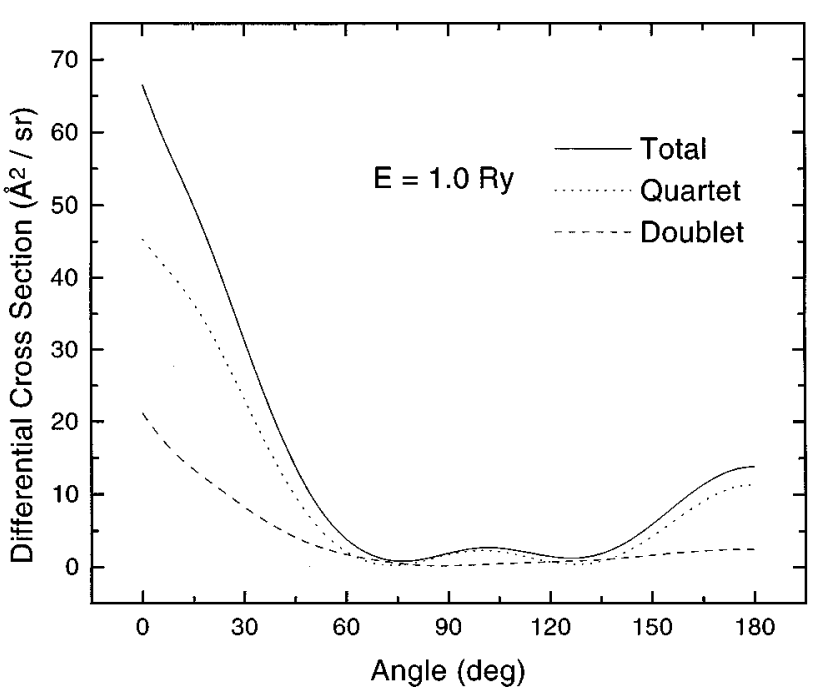

FIG. 4. Differential cross section for elastic scattering of electrons from sulfur at energy $E=1.0 \mathrm{Ry}$ as a function of scattering angle. 


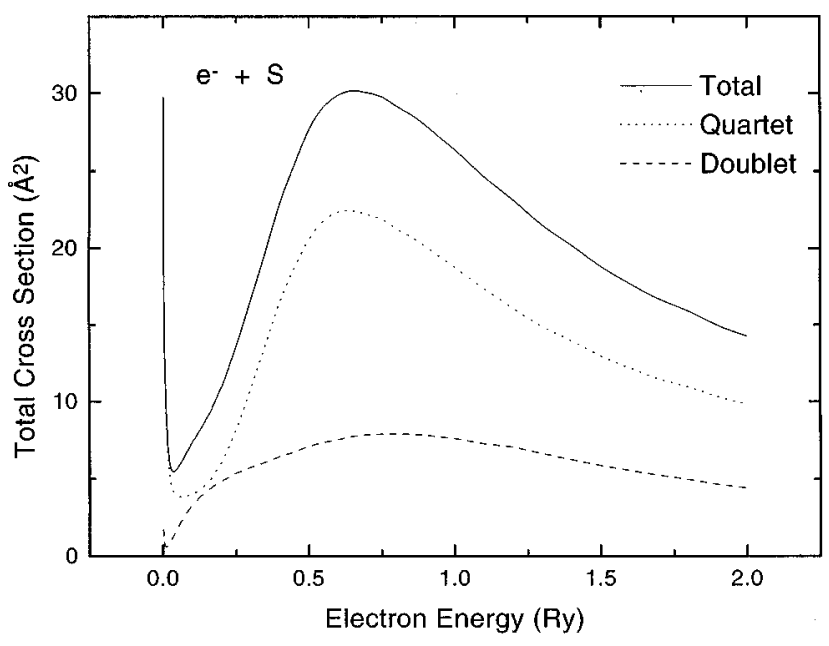

FIG. 5. Total cross sections for elastic scattering of electrons from sulfur as a function of kinetic energy.

ergies the quartet contributions are much larger than those for the doublet. The quartet and total cross sections for energy 0.01 Ry decrease more rapidly than those for energy $0.001 \mathrm{Ry}$.

It is seen from Fig. 3 that for energy $0.1 \mathrm{Ry}$, the quartet cross section initially decreases with increasing angle of scattering, passes through a minimum around $50^{\circ}$, then oscillates slowly with small amplitude. However, the doublet cross section initially increases, passes through a maximum at about $60^{\circ}$, and then decreases. For small angles, the quartet cross section is much larger than that of the doublet; between $40^{\circ}$ and $100^{\circ}$ it is smaller and for higher angles it is again larger. The total cross section has a local minimum at about $45^{\circ}$ and a local maximum at about $70^{\circ}$. For energy $1.0 \mathrm{Ry}$, as shown in Fig. 4, the quartet and total cross sections essentially follow each other. Each has two minima at about $75^{\circ}$ and $125^{\circ}$, whereas the doublet is smaller and has a minimum at about $90^{\circ}$.

\section{E. Total cross sections}

The total cross sections are shown in the Fig. 5 as a function of incident electron energy from 0.0 to $2.0 \mathrm{Ry}$. From the figure it is seen that the Ramsauer-Townsend minima occur at around 0.06 and $0.01 \mathrm{Ry}$ for the quartet and doublet cross sections, respectively. The total cross section has the minimum at $0.04 \mathrm{Ry}$. The magnitudes of the minima for quartet and doublet cross sections are $3.848 \AA^{2}$ and $0.598 \AA^{2}$, respectively, whereas for the total the magnitude is $5.489 \AA^{2}$.

\section{F. Momentum-transfer cross sections}

The present momentum-transfer cross sections for the quartet and doublet terms, and the sum of the two, are plotted in Fig. 6 as a function of incident energy ranging from 0.0 to

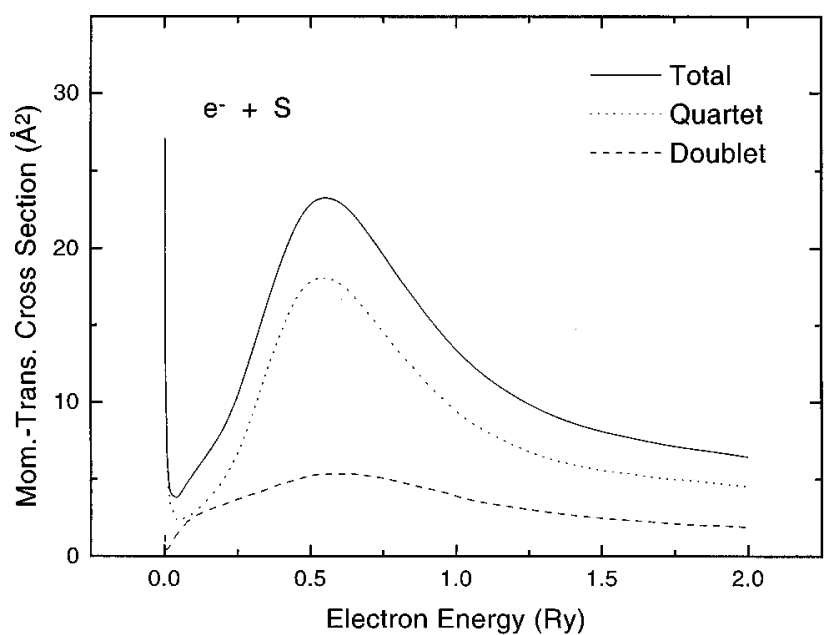

FIG. 6. Momentum-transfer cross sections for elastic scattering of electrons from sulfur as a function of kinetic energy.

2.0 Ry. The Ramsauer-Townsend minima for the doublet, quartet, and total momentum-transfer cross sections occur respectively at $0.008,0.05$, and $0.04 \mathrm{Ry}$. The magnitudes of these minima are $0.409,2.304$, and $3.750 \AA^{2}$, respectively.

\section{CONCLUSION}

Very careful and accurate calculations have been performed on the low-energy elastic scattering of electrons from sulfur atoms using the multiconfiguration Hartree-Fock method for continuum wave functions. The electron correlation and polarization effects, which are extremely important for the case of open-shell atoms, have been taken into account in an $a b$ initio way through the configurationinteraction procedure. The scattering length is computed completely $a b$ initio with the wave function calculated exactly at zero energy. The magnitude and position of the Ramsauer-Townsend minimum, which is the most interesting feature in the case of elastic scattering of electrons from atoms, has been determined. The present MCHF method has the advantage that the energy-dependent polarization and electron correlation effects are obtained accurately by optimizing both excited bound and scattering electron wave functions simultaneously at each kinetic energy of the scattered electron. As there is no experimental measurement available for this elastic scattering of electrons from sulfur atoms, it is hoped that the present theoretical results will be useful in stimulating such experiments.

\section{ACKNOWLEDGMENTS}

I wish to thank Dr. I. I. Fabricant for suggesting the calculations. This research was supported in part by the $\mathrm{Na}-$ tional Science Foundation.
[1] I. I. Fabricant, J. Phys. B 27, 4545 (1994).

[2] H. P. Saha, Phys. Rev. A 47, 273 (1993).

[3] H. P. Saha, Phys. Rev. A 43, 4712 (1991).

[4] A. Dasgupta and A. K. Bhatia, Phys. Rev. A 32, 3335 (1985).
[5] R. P. McEachran and A. D. Stauffer, J. Phys. B 16, 4023 (1983).

[6] R. P. McEachran and A. D. Stauffer, J. Phys. B 17, 2507 (1984). 
[7] R. P. McEachran and A. D. Stauffer, J. Phys. B 20, 3483 (1987).

[8] H. P. Saha, Phys. Rev. A 53, 1553 (1996).

[9] H. P. Saha (unpublished).

[10] R. K. Nesbet, Adv. At. Mol. Phys. 13, 315 (1977).

[11] Frank E. Harris and H. Michels, Methods Comput. Phys. 10, 143 (1971).

[12] J. M. Blatt and L. C. Biedenharn, Rev. Mod. Phys. 24, 258 (1952).

[13] L. C. Biedenharn, J. M. Blatt, and M. E. Rose, Rev. Mod. Phys. 24, 249 (1952).
[14] C. Froese Fischer, Comput. Phys. Commun. 14, 145 (1978).

[15] T. F. O’Malley, L. Spruch, and L. Rosenberg, J. Math. Phys. (N.Y.) 2, 491 (1961).

[16] H. P. Saha and C. D. Caldwell, Phys. Rev. A 44, 5642 (1991).

[17] H. P. Saha, Phys. Rev. A 47, 2865 (1993).

[18] T. M. Miller and B. Bederson, Adv. At. Mol. Phys. 13, 1 (1977).

[19] H. P. Saha, Phys. Rev. A 48, 1163 (1993).

[20] H. P. Saha, Phys. Rev. Lett. 65, 2003 (1994). 